\title{
Perilipin 1 Expression Differentiates Liposarcoma from Other Types of Soft Tissue Sarcoma
}

Beate K. Straub, ${ }^{\star \dagger}$ Hagen R. Witzel, ${ }^{*}$ Lena M. Pawella, ${ }^{\dagger}$ Marcus Renner, ${ }^{\dagger}$ Eva Eiteneuer, ${ }^{\dagger}$ Merita Hashani, ${ }^{\dagger \dagger}$ Peter Schirmacher, Wilfried Roth, ${ }^{*}$ and Gunhild Mechtersheimer

From the Institute of Pathology, ${ }^{*}$ University Medical Center Mainz, Mainz, Germany; the Department of General Pathology ${ }^{\dagger}$ Institute of Pathology, University Hospital, Heidelberg, Germany; and the Institute of Pathology, ${ }^{\ddagger}$ University Hospital of Prishtina, Prishtina, Kosovo

\author{
Accepted for publication \\ April 10, 2019. \\ Address correspondence to \\ Beate K. Straub, M.D., Institute \\ of Pathology, University Medi- \\ cal Center Mainz, Langenbeck- \\ strasse 1, D-55131 Mainz, \\ Germany. E-mail: beate.straub \\ @ unimedizin-mainz.de.
}

\begin{abstract}
Lipid droplets, a morphologic feature of adipocytic tumors, are strongly regulated by associated proteins of the perilipin/PAT (perilipin, adipophilin, and tail-interacting protein of $47 \mathrm{kD}$ ) family. So far, the use of perilipins as markers for differential diagnosis of soft tissue tumors has only been studied in a few cases. The aim of this study was to investigate the expression of perilipins in 478 human soft tissue tumors and 60 respective normal tissues. Perilipin 1 was immunohistochemically positive in all studied cases of well-differentiated liposarcomas, $>90 \%$ of myxoid round cell liposarcomas, and $>70 \%$ of pleomorphic liposarcomas, whereas only the differentiated components of dedifferentiated liposarcomas were immunohistochemically positive for perilipin 1. All other types of soft tissue sarcomas were negative for perilipin 1. Perilipin 2 was more prominent in dedifferentiated and pleomorphic liposarcomas and nearly all other high-grade sarcomas. In well-differentiated liposarcomas, lipomas, or normal adipose tissue, perilipin 2 was virtually absent. In addition, long-term stimulation of adipogenesis in the liposarcoma cell line LiSa-2 restored perilipin 1 expression, as exhibited in the source tumor. Furthermore, knockdown of perilipin 2 or perilipin 3 in LiSa-2 cells influenced lipid droplet number and size as well as cell vitality. In summary, perilipin 1 is a promising marker for the differential diagnosis of liposarcomas from other soft tissue sarcomas, whereas perilipin 2 correlates negatively with tumor grade and may be therapeutically useful. (Am J Pathol 2019, 189: 1547-1558; https:// doi.org/10.1016/j.ajpath.2019.04.017)
\end{abstract}

Lipid droplets (LDs) are ubiquitous cytoplasmic cell organelles. They consist of a core of triacylglycerides and cholesterol ester, covered by a phospholipid monolayer. The composition of proteins found in LDs is highly variable, depending on the cell type or specific status of the cell, and strongly regulates lipid storage and release. The most abundant proteins found to be embedded in the monolayer belong to the amphiphilic perilipin family [formerly named the PAT family after the first three constituents: perilipin, adipophilin, and tail-interacting protein of $47 \mathrm{kD}$ (TIP47); concerning actual nomenclature ${ }^{1}$ ]. In mammals, the perilipin family consists of five members: perilipin 1 (formerly perilipin ${ }^{2}$ ), perilipin 2 (adipophilin, adipose differentiation-related protein, and $\left.\mathrm{ADFP}^{3-5}\right)$, perilipin $3\left(\mathrm{TIP}_{4} 7^{6,7}\right)$, perilipin 4 $\left(\mathrm{S} 3-12^{8}\right)$, and perilipin 5 (myocardial lipid droplet protein,
OXPAT, and lipid storage droplet protein $5^{9,10}$ ). Perilipins tightly regulate $\mathrm{LD}$ biogenesis and degradation and are required to maintain their structural integrity. In differentiating adipocytes, perilipins are recruited to LDs in a highly coordinated manner. ${ }^{11}$ Perilipins 3,4 , and 5 form a cytosolic pool and translocate to nascent LDs, whereas perilipins 1 and 2 directly associate with mainly larger LDs after being expressed. ${ }^{8,9,12,13}$ Mature LDs of adipocytes are covered exclusively by perilipin $1,{ }^{3}$ which is responsible for long-

Supported, in part, by German Research Foundation grants STR 1160/1 2 (B.K.S.). M.H. held a stipend of the Erasmus Basileus-Program, and B.K.S. held a stipend of the Olympia-Morata program of the Medical Faculty of Heidelberg University.

Disclosures: None declared. 
term storage of neutral lipids and their coordinated degradation on adrenergic stimuli. Therefore, perilipin 1 exhibits specific function in adipocytes of white and brown adipose tissue, in steatotic hepatocytes, and in sebaceous gland epithelial cells. ${ }^{2,14-16}$ Knockout of Plin1 in mice leads to a lean phenotype caused by largely reduced mature adipose tissue, even under a high-fat diet or in leptin deficiency. ${ }^{17,18}$ However, in humans, perilipin 1 deficiency leads to autosomal dominant partial lipodystrophy, hepatocyte steatosis, and high blood triacyl glycerol levels. ${ }^{19}$ In contrast to the limited perilipin 1 expression, perilipins 2 and 3 are ubiquitously expressed. ${ }^{3,4,7}$ Perilipin 5 is highly expressed in tissues with oxidative energy supply, such as the heart, the liver after fasting, skeletal muscle, and brown adipose tissue, ${ }^{9,10}$ regulating b-adrenoceptor-stimulated lipolysis via adipose triglyceride lipase $\mathrm{e}^{20,21}$ as well as in differentiating adipocytes. ${ }^{13}$ Perilipin 4 is predominantly found in adipocytes, , $^{8,16,22}$ whereas its role is widely understudied so far.

Adipocytic tumors represent the largest single group of mesenchymal tumors, and liposarcoma is the single most common type of soft tissue sarcoma. To date, four subtypes of liposarcoma are distinguished: well- differentiated liposarcoma (WDLS)/atypical lipomatous tumor, dedifferentiated liposarcoma (DDLS), myxoid/round cell liposarcoma (MLS), and pleomorphic liposarcoma (PLS). Apart from the morphologically characteristic lipoblast, immunohistochemistry with positivity for S100 may help in pathologic diagnosis. WDLS and DDLS are genetically defined by supernumerary ring chromosomes containing amplifications in mouse double minute 2 homolog (MDM2). MLSs are characterized by a fusion of DNA damage inducible transcript 3 [DDIT3; synonym: CCAATenhancer-binding protein homologous protein $(\mathrm{CHOP})]$ and fused in sarcoma/translocated in sarcoma (FUS/TLS); thus, molecular pathology supports histopathologic diagnosis in these subtypes. ${ }^{23}$ PLS, however, harbors no characterizing molecular aberrations. Although WDLS, MLS, and infrequently also PLS show lipoblasts with LDs visible in conventional light microscopy, LDs are rare in dedifferentiated components of DDLS.

Although localization and function of perilipins have been extensively analyzed during adipogenesis in animal and cell culture models, the role of perilipins in liposarcoma remains largely unexplored. ${ }^{24,25}$ Therefore, we analyzed the expression and localization of perilipins 1 through 5 to determine their potential use as markers for the differential diagnosis of liposarcoma and as therapeutic targets.

\section{Materials and Methods}

\section{Tissues and Cultured Cells}

Cryopreserved and formalin-fixed, paraffin-embedded human tissue samples were provided by the tissue bank of the National Center for Tumor Diseases (Heidelberg, Germany) in accordance with the ethics committee of the University of Heidelberg (Heidelberg, Germany; numbers 206/2005 and 207/2005). In detail, a multitissue array was used that contained 478 soft tissue tumors and 60 normal tissue samples. All cores were present in duplicate (Supplemental Table S1 and Table 1). Diagnoses were based on standard histopathologic, immunohistologic, and molecular pathologic criteria of the current eighth World Health Organization classification. For protein analysis and immunofluorescence studies, sarcoma tissues were frozen in liquid nitrogen and kept at $-80^{\circ} \mathrm{C}$ until needed. Cells of the liposarcoma cell line LiSa- $2,{ }^{26}$ which was derived from a pleomorphic liposarcoma, and T778 cells, derived from a well-differentiated liposarcoma, were cultured in RPMI 1640 medium supplemented with $10 \%$ fetal calf serum and

Table 1 Positivity for Perilipin 1 in Soft Tissue Tumors

\begin{tabular}{lcc}
\hline Soft tissue tumor & Number of cases & Perilipin 1 LD-expression, \% \\
\hline WDLS & 24 & 100 \\
DDLS & 48 & $0-38$ \\
MLS & 30 & 97.3 \\
PLS & 13 & 72.7 \\
Lipoma or angiolipoma & 15 & 100 \\
EWS & 18 & 0 \\
GIST & 6 & 0 \\
Fibroblastic tumors (eg, myxofibrosarcoma and fibromatosis) & 65 & 0 \\
Muscle tumors (eg, leiomyosarcoma and rhabdomyosarcoma) & 70 & 0 \\
Nerve sheath tumors (eg, MPNST, neurofibroma, and & 52 & 0 \\
$\quad$ schwannoma) & & 0 \\
Neural tumors (eg, neuroblastoma and ganglioneuroma) & 38 & 0 \\
Tumors of uncertain differentiation (eg, synovial sarcoma) & 40 & 0 \\
Undifferentiated pleomorphic sarcoma & 63 & 0
\end{tabular}

Immunohistochemical analysis of soft tissue tumors with an antibody against perilipin 1 . The percentage of cases that showed perilipin 1 expression at LDs was determined. All cases were present in duplicate in the tumor tissue microarray.

*For cytoplasmic staining of perilipin 1 in ganglion cells in ganglioneuroblastoma, see Supplemental Figure S2.

DDLS, dedifferentiated liposarcoma; EWS, Ewing sarcoma; GIST, gastrointestinal tract stroma tumor; LD, lipid droplet; MLS, myxoid liposarcoma; MPNST, malignant peripheral nerve sheath tumor; PLS, pleomorphic liposarcoma; WDLS, well-differentiated liposarcoma. 
$1 \%$ penicillin-streptomycin (100 $\mathrm{U} / \mathrm{mL})$, as described previously. ${ }^{27}$

\section{siRNA Knockdown}

siRNA-mediated knockdown of perilipins 2 and 3 was performed, as previously described $.^{28} \mathrm{LiSa}-2$ cells were seeded in a $24-w e l l$ plate ( 4000 cells per well), and siRNAs $(10 \mathrm{nmol} /$ L, final concentration) were transfected the day after with transfection reagent (Oligofectamine; Invitrogen, Carlsbad, $\mathrm{CA}$ ), according to the manufacturer's specifications. The double knockdown was performed with $5 \mathrm{nmol} / \mathrm{L}$ of each siRNA. Cells were collected after 48 or 72 hours and further subjected to immunostaining, immunoblot analysis, and the MTT assay. siRNA sequences were as follows: perilipin 2 number 1, 5'-GCACAUUGAGUCACGUACU(dUdU)- $3^{\prime}$ (forward) and 5'-AGTACGTGACTCAATGTGC(dUdU)-3' (reverse); perilipin 2 number 2, 5'-GAAGCAAGUAGCUAGUAUA(dUdU)-3' (forward) and 5'-TATACTAGCTACTTGCTTC(dUdU)-3' (reverse); perilipin 3 number $1,5^{\prime}$-GAACAGAGCUACUUCGUAC(dUdU)- $3^{\prime}$ (forward) and $5^{\prime}$-GTACGAAGTAGCTCTGTTC(dUdU)-3' (reverse); perilipin 3 number $2,5^{\prime}$-GUCCUAAGCCUGAUGGAAA(dUdU)- $3^{\prime}$ (forward) and 5'-TTTCCATCAGGCTTAGGAC(dUdU)- $3^{\prime}$ (reverse); nontargeting number $1,5^{\prime}$ UUCUCCGAACGUGUCACGU(dUdU)-3' (forward) and 5'-ACGTGACACGTTCGGAGAA(dUdU)-3' (reverse); nontargeting number $2,5^{\prime}$-CUUACUCUCGCCCAAGCGA(dUdU)-3' (forward) and 5'-TCGCTTGGGCGAGAGTAAG(dUdU)-3'(reverse); and nontargeting number $3,5^{\prime}$ UGGUUUACAUGUCGACUAA(dUdU)- $3^{\prime}$ (forward) and 5'-TTAGTCGACATGTAAACCA(dUdU)-3' (reverse).

\section{Cell Viability Assay (MTT Assay)}

LiSa- 2 cells were seeded in a 24-well plate (4000 cells per well) and transfected the day after with transfection reagent, according to the manufacturer's specifications. After 48 or 72 hours, the culture medium was replaced with culture medium supplemented with MTT assay and incubated for 1 hour at $37^{\circ} \mathrm{C}$. Afterward, the medium was removed, the formazan crystals were solved in dimethyl sulfoxide/ethanol (1:2), and absorbance was measured at 570-nm wavelength. The viability in control cells (nontransfected) was set to $100 \%$. The assay was repeated with 1000 or 3000 cells seeded per well under equal conditions, showing comparable results (data not shown).

\section{Antibodies and Reagents}

Guinea pig polyclonal antibodies against perilipin 1 (GP29; dilution 1:1000), perilipin 2 (GP40; dilution 1:1000), perilipin 3 (GP30; dilution 1:1000), perilipin 4 (GP38; dilution 1:1000), and perilipin 5 (GP31; dilution 1:1000) were from Progen Biotechnik (Heidelberg, Germany). Mouse monoclonal antibodies against perilipin 1 (Peri 112.17; dilution 1:100) and perilipin 2 (clone AP125; dilution 1:100) were from Progen Biotechnik, and mouse monoclonal antibodies against actin (C4; dilution 1:10.000) were from Millipore (Billerica, MA). In addition, rabbit polyclonal antibodies against $\mathrm{N}$-terminal amino acid 10 through 29 (Plin5 1.1 and 1.2; dilution 1:100) and against central amino acid 254 through 296 (Plin5 2.1 and 2.2; dilution 1:100) of perilipin 5 were generated by Thermo Fisher Scientific (Bremen, Germany ${ }^{29}$ ).

\section{Immunofluorescence Microscopy}

Snap-frozen and cryopreserved human tissue samples were cut with a cryostat to a thickness of $5 \mu \mathrm{m}$ and fixed afterward with either methanol and acetone at $-20^{\circ} \mathrm{C}$ (each, 5 minutes) or, in the case where preservation of lipids was required, with $4 \%$ paraformaldehyde at $4{ }^{\circ} \mathrm{C}$ for 10 minutes. Immunofluorescence staining was performed, as previously described. ${ }^{14,30}$ Sections were incubated with primary antibodies in phosphate-buffered saline (PBS) for 30 minutes, washed with PBS (twice for 5 minutes), and subsequently incubated with secondary antibodies in PBS for 30 minutes. This was followed by two washing steps with PBS for 5 minutes and with double-distilled water for $<1$ second. Sections were mounted with Fluoromount G (Biozol Diagnostica, Eching, Germany). Stained tissue sections were analyzed by confocal A1R laser scanning microscopy (Nikon, Duesseldorf, Germany).

\section{Immunohistochemistry of Formalin-Fixed, Paraffin-Embedded Tissue}

Immunohistochemistry using formalin-fixed, paraffinembedded tissues was performed as described previously. ${ }^{14,31,32}$ Formalin-fixed, paraffin-embedded tissue sections $(2 \mu \mathrm{m}$ thick) were deparaffinized with xylene (three times for 10 minutes) and rehydrated with an ethanol gradient. Antigen retrieval was performed with 5\% urea in $0.1 \mathrm{~mol} / \mathrm{L}$ Tris- $\mathrm{HCl}\left(\mathrm{pH} \mathrm{9.5)} \mathrm{for} 15\right.$ minutes at $100^{\circ} \mathrm{C}$. Afterward, the sections were incubated with $3 \% \mathrm{H}_{2} \mathrm{O}_{2}$ in PBS for 30 minutes, washed with PBS (twice for 5 minutes), and blocked with $10 \%$ of the respective serum, avidin, and 5\% milk powder in PBS for 30 minutes. The samples were washed with PBS (twice for 5 minutes) and blocked with $10 \%$ of the respective serum, biotin, and $5 \%$ milk powder in PBS for 30 minutes. Primary antibodies were applied for 1 hour or overnight. Sections were washed with PBS (twice for 5 minutes). Secondary antibodies were applied for 30 minutes. After subsequent washes with PBS, the sections were incubated with the avidin-biotin complex for $30 \mathrm{mi}-$ nutes. After washing with PBS, sections were incubated with diaminobenzidine substrate for 2 to 10 minutes. The reaction was stopped with double-distilled water.

Counterstaining of the nuclei was performed with hematoxylin-eosin solution (15 to 20 seconds).

Sections were mounted with Eukitt (Riedel de Haen, Seelze, Germany). 


\section{Gel Electrophoresis and Immunoblotting}

Frozen human tissue sections were lysed using cell lysis buffer (Cell Signaling Technology, Frankfurt, Germany), including a protease inhibitor cocktail (Sigma, Deisenhofen, Germany). Protein concentration was measured with a Bradford assay (Bio-Rad, Munich, Germany). Protein extracts were dissolved in Laemmli buffer and separated by SDS-PAGE. After transfer of separated proteins onto polyvinylidene difluoride membranes (Bio-Rad), membranes were blocked with $5 \%$ milk powder in Tris-buffered saline with Tween 20 at room temperature for 1 hour. Membranes were incubated with the respective primary antibodies diluted in blocking solution and incubated overnight at $4^{\circ} \mathrm{C}$. After washing the membrane in Tris-buffered saline with Tween 20, three times for 10 minutes, horseradish peroxidase-coupled secondary antibodies were also diluted in blocking solution added and incubated at room temperature for 1 hour. Immunodetection was performed after additional washing, at room temperature with Tris-buffered saline with Tween 20, three times for 10 minutes, and then blots were developed with the chemiluminescence reagent electrochemiluminescence kit (PerkinElmer, Waltham, MA).

\section{Results}

\section{Differential Expression of Perilipins in Adipocytic Tumors}

Although extensive studies on perilipins have been performed in adipocytes, few studies have been performed in liposarcoma. Therefore, the expression of perilipin 1 through 5 and their localization in human soft tissue tumors were studied using a tissue microarray (Supplemental Table S1), including 478 soft tissue tumors and 60 representative normal tissues (Figure 1). Immunohistochemical analysis of normal human white adipose tissue (WAT) showed strong staining for perilipins 1 and 4, whereas perilipins 2, 3, and 5 were virtually negative (Figure 1A). Only vessels or foam cell macrophages showed staining for perilipins 2,3 , and 5 . In brown adipose tissue, independent of the patient age, staining was detected for all perilipins (Figure 1A). Perilipin 1 was positive at large LDs in normal adipose tissue (26/26), all benign lipogenic tumors (15/15), WDLSs (49/49), and the vast majority of MLSs $(56 / 62=90 \%)$ and PLSs (14/ $20=70 \%$ ), whereas perilipin 1 was only detected in a few small LDs in DDLSs (35/80) (Figure 1B, Supplemental Table S1, and Supplemental Figure S1). Staining for perilipin 1 revealed, especially in DDLSs, MLSs, and PLSs, small LDs not visible in conventional hematoxylin and eosin-stained sections (for DDLSs) (Supplemental Figure S1) and may thereby be of diagnostic help in identifying lipogenic differentiation.

In adipocytic tumors, perilipin 4 expression was similar to perilipin 1, yet not restricted to liposarcomas. In contrast, perilipins 2 and 3 were absent in lipomas and only expressed at low levels in WDLSs, but demonstrated increased numbers of LDs in PLSs, MLSs, and DDLSs (Figure 1B). Especially, lipoblasts showed positivity for perilipins 2 and 3 besides the expression of other perilipins. Yet, some lipoma specimens with pronounced inflammatory changes showed high perilipin 2 staining, especially in foam cell macrophages. Perilipin 5, however, was expressed in all adipocytic tumors to a relatively equal extent, with MLSs and PLSs showing the highest expression. Along the liposarcoma subtypes, MLSs and PLSs demonstrated the highest expression of all perilipins. WDLSs showed the highest amounts of perilipins 1 and 4 and the lowest amounts of perilipins 2 and 3, whereas the lowest amounts of perilipins 1 and 4 and the highest amounts of perilipins 2 and 3 were found in DDLSs.

In parallel, the total protein obtained from WAT, lipomas, and different types of liposarcomas (WDLSs, DDLSs, MLSs, and PLSs) was analyzed for the presence of perilipins 1 through 5 by immunoblot. Perilipin 1 expression was detected in WAT, lipomas, and most cases of WDLSs, but was reduced in MLSs and PLSs. The dedifferentiated components of all cases of DDLS analyzed were negative for perilipin 1 (Figure 2A). Perilipins 4 and 5 showed an almost similar expression pattern compared with perilipin 1 , with perilipin 4 expression in the WAT and MLS samples and perilipin 5 expression in all MLS samples analyzed. In contrast, perilipin 2 and perilipin 3 expression was found in all cases in WAT, most likely because of the presence of nonadipocytic cells.

Immunofluorescence microscopy of the same cases was performed using boron dipyrromethene (BODIPY) to emphasize the presence of LDs in liposarcoma. BODIPYpositive LDs were detected in WDLSs, MLSs, and PLSs, but only sparse and minute LDs were detected in the dedifferentiated components of DDLSs (Figure 2B). Perilipin 1 surrounded LDs, yet differentially with respect to perilipin 2 .

In summary, perilipins 1 through 5 exhibited differential expression patterns in adipocytic tumors, perilipins 1 and 4 exhibited higher levels in better-differentiated tumors and negatively correlated with tumor grade, and perilipins 2,3 , and 5 were expressed in higher levels in less-differentiated tumors (Supplemental Table S2).

\section{Perilipins in Sarcomas Other than Liposarcomas}

In contrast to adipogenic tumors, perilipin 1 was absent from all other soft tissue sarcoma tumors, including Ewing sarcomas, myxofibrosarcomas, desmoid-type fibromatoses, gastrointestinal tract stroma tumors, leiomyosarcomas, rhabdomyosarcomas, malignant peripheral nerve sheath tumors, neuroblastomas, synovial sarcomas, and undifferentiated pleomorphic sarcomas (Table 1, Supplemental Tables S1 and S3, and Figure 3A). Faint nuclear staining for perilipin 1 was detected in some high-grade sarcomas (Supplemental Figure S2). In addition, vegetative ganglion and ganglioneuroblastomas showed faint cytoplasmic 


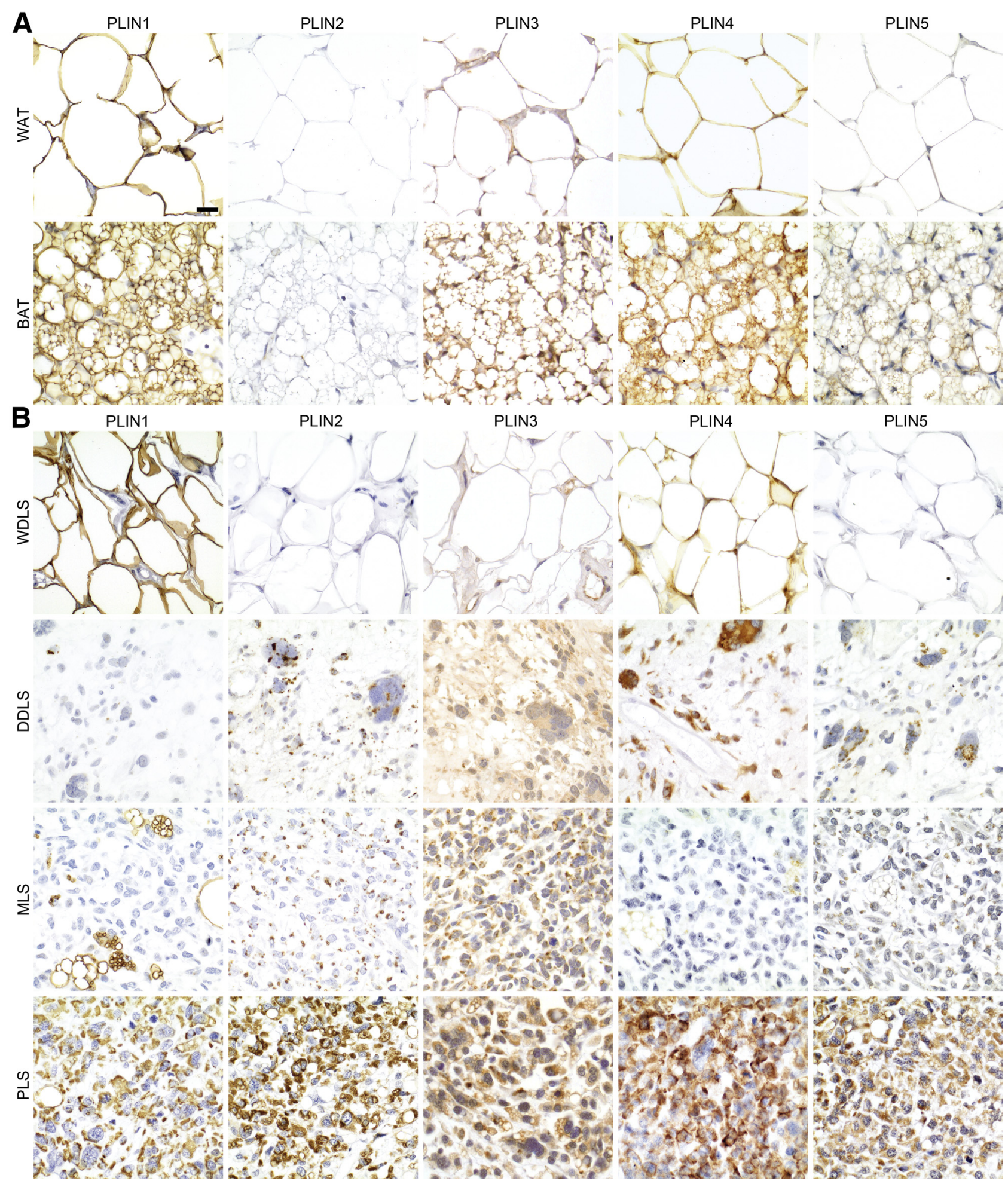

Figure 1 Immunohistochemical analysis of perilipins 1 through 5 (PLIN1-5) in liposarcoma. A: Perilipins were differentially expressed in normal white (WAT) and brown (BAT) adipose tissue. B: Expression for virtually all perilipins (PLIN1-5) was detected in well-differentiated liposarcoma (WDLS), dedifferentiated liposarcoma (DDLS), myxoid liposarcoma (MLS), and pleomorphic liposarcoma (PLS). Scale bar $=20 \mu \mathrm{m}$.

staining for perilipin 1; however, this occurred without definitive staining of LDs (Supplemental Figure S3). Perilipins $2,3,4$, and 5 were expressed virtually ubiquitously with cell type-specific differences (Figure 3A). Perilipin 2 expression was especially high in high-grade sarcomas, such as in undifferentiated pleomorphic sarcomas, and rather low in benign tumors. In line with this finding, smooth muscle and leiomyomas showed no or only faint staining for 


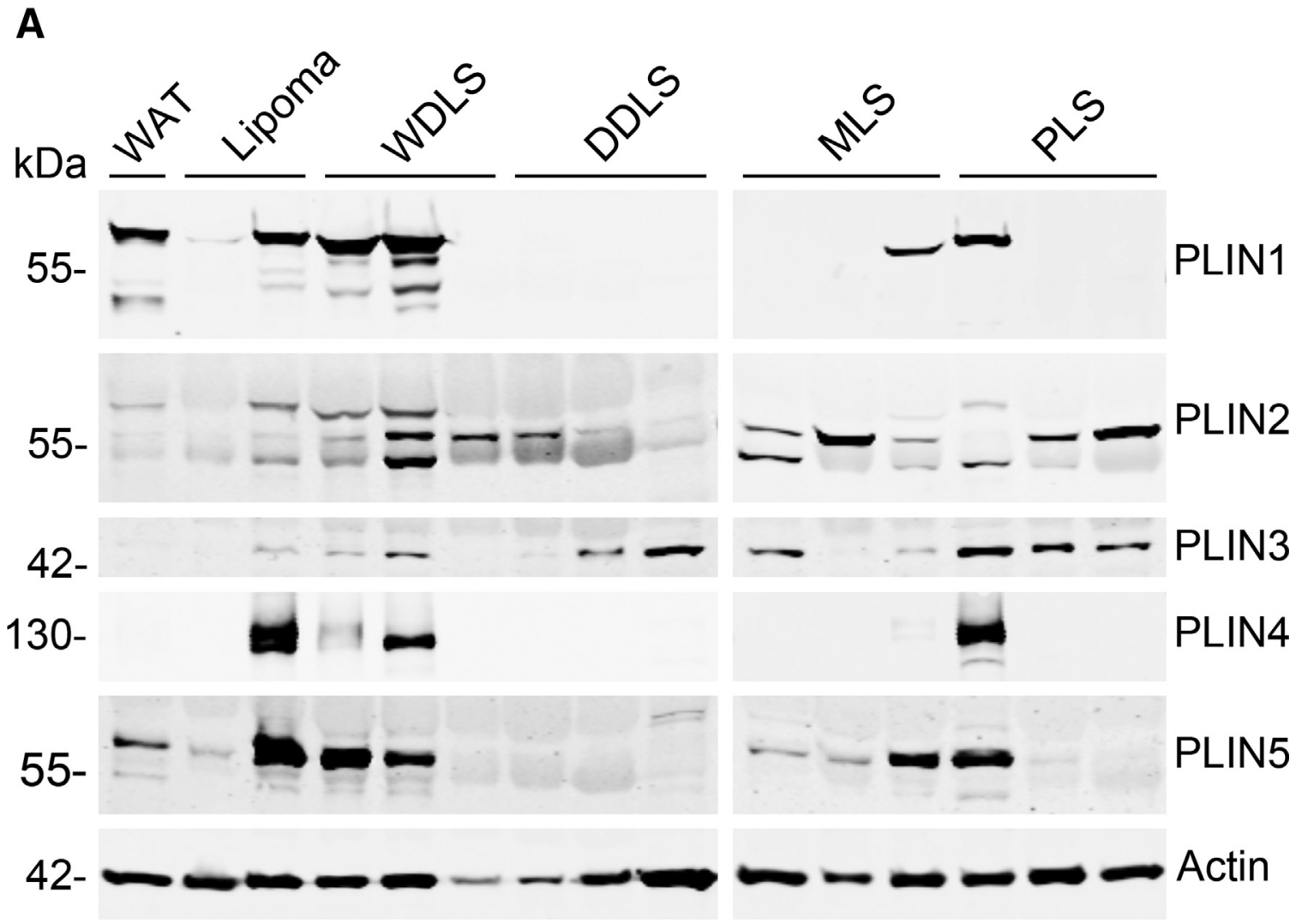

B
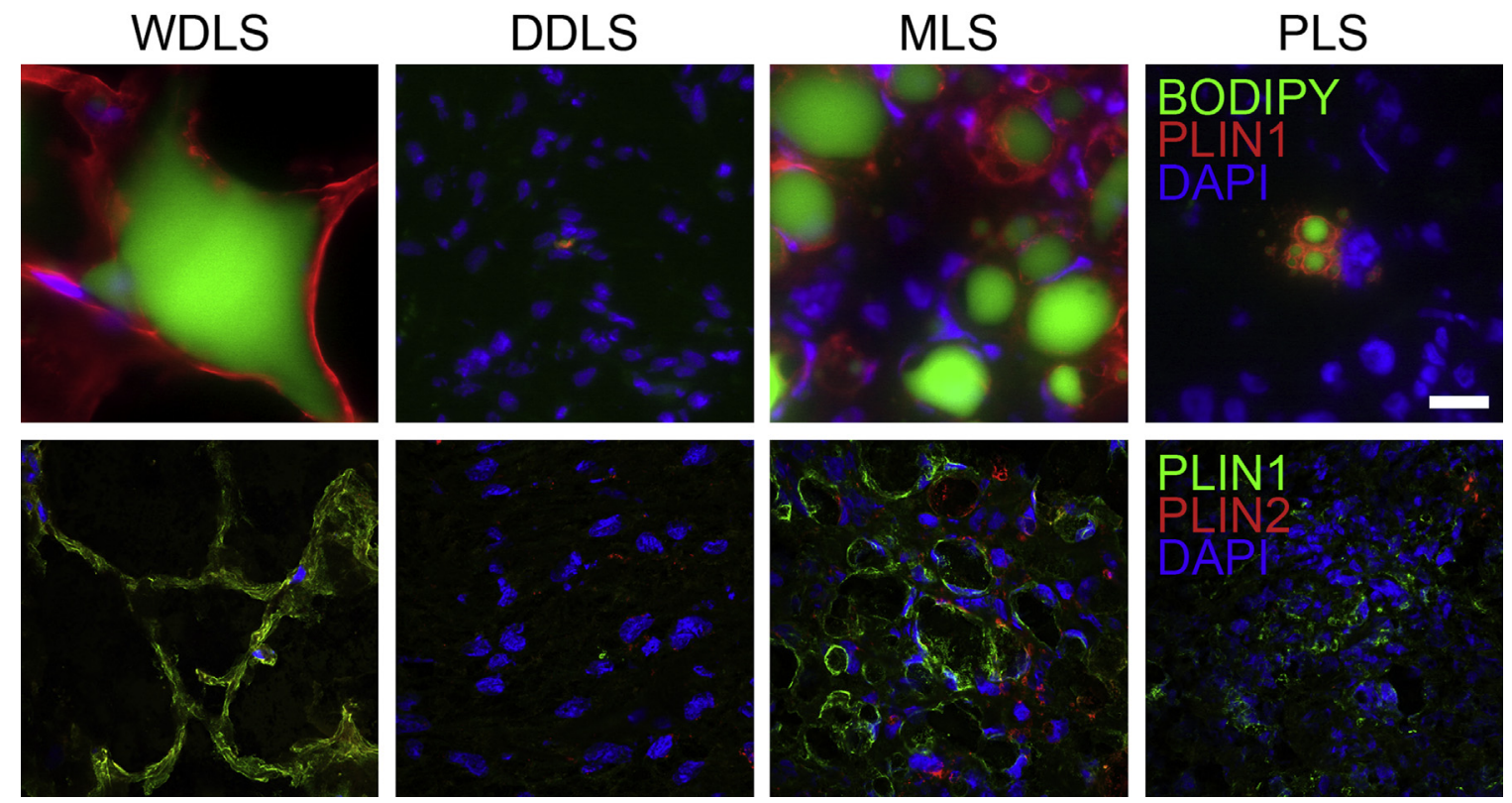

Figure 2 Perilipins 1 through 5 (PLIN1 through PLIN5) characterize liposarcoma. A: Immunoblot analysis of whole tissue lysates of adipose tissue, lipoma, and liposarcoma samples: white adipose tissue (WAT; $1 \times$ ), lipoma $(2 \times)$, and liposarcoma [well-differentiated liposarcoma (WDLS), dedifferentiated liposarcoma (DDLS), myxoid liposarcoma (MLS), and pleomorphic liposarcoma (PLS); each $3 \times$ ] with antibodies against PLIN1 through PLIN5. Most WDLSs and some cases of MLS and PLS show expression for all perilipins. In DDLS, however, perilipins 1 and 5 are absent and perilipins 2 and 3 are present at low amounts. Actin served as the protein control. Molecular weight marker is shown on the left. B: Immunofluorescence analysis of WDLS, DDLS, MLS, and PLS sections stained with antibodies against PLIN1 and PLIN2. Lipid droplets (LDs) were stained with boron dipyrromethene (B0DIPY). Nuclei were stained with DAPI. In WDLS and MLS sections, PLIN1 localizes at LDs, whereas only focal expression is present in DDLS and PLS. Note differential expression of PLIN1 (green) and PLIN2 (red) in liposarcoma. Scale bar $=20 \mu \mathrm{m}$. 
A

$\square$ Normal tissue $\square$ Tumors (benign) $\square$ Tumors (malignant)

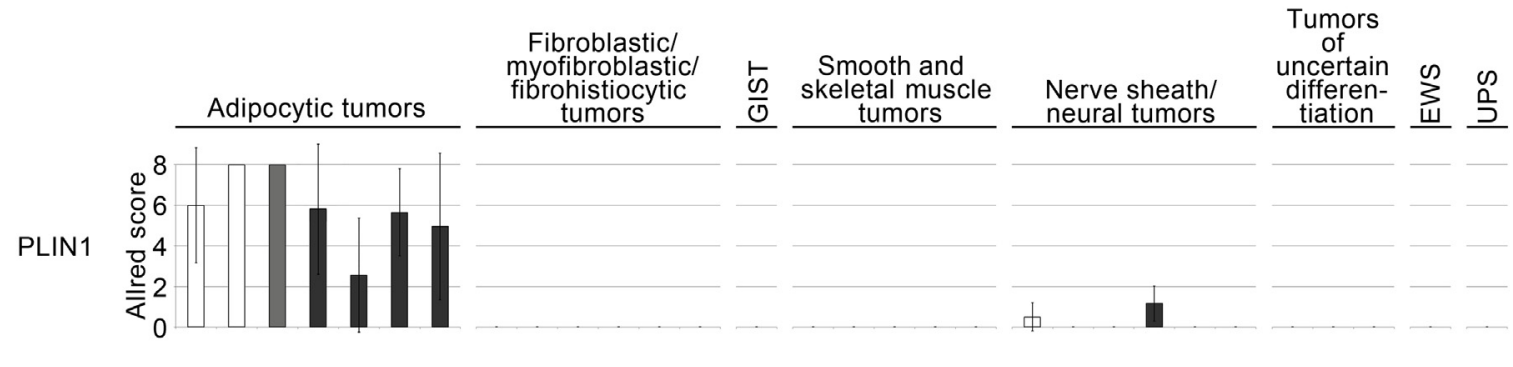

PLIN2

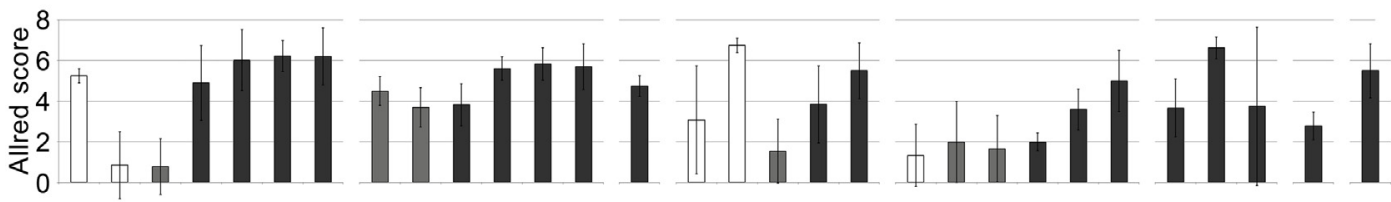

PLIN3

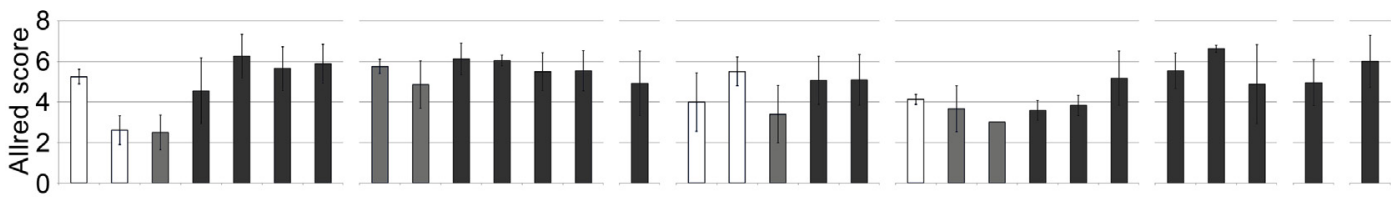

PLIN4

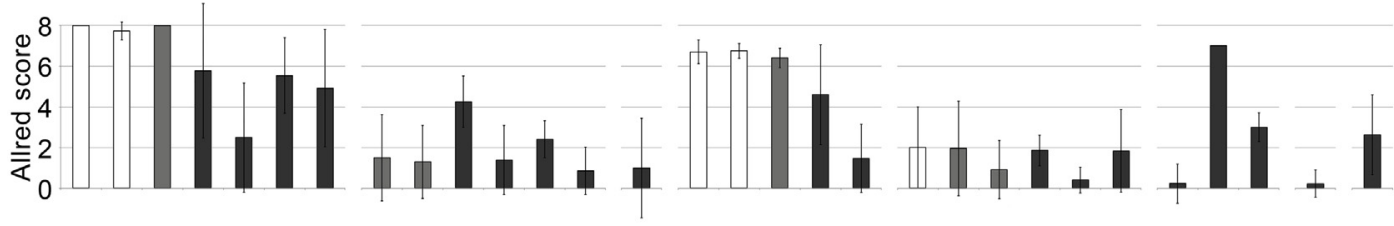

PLIN5
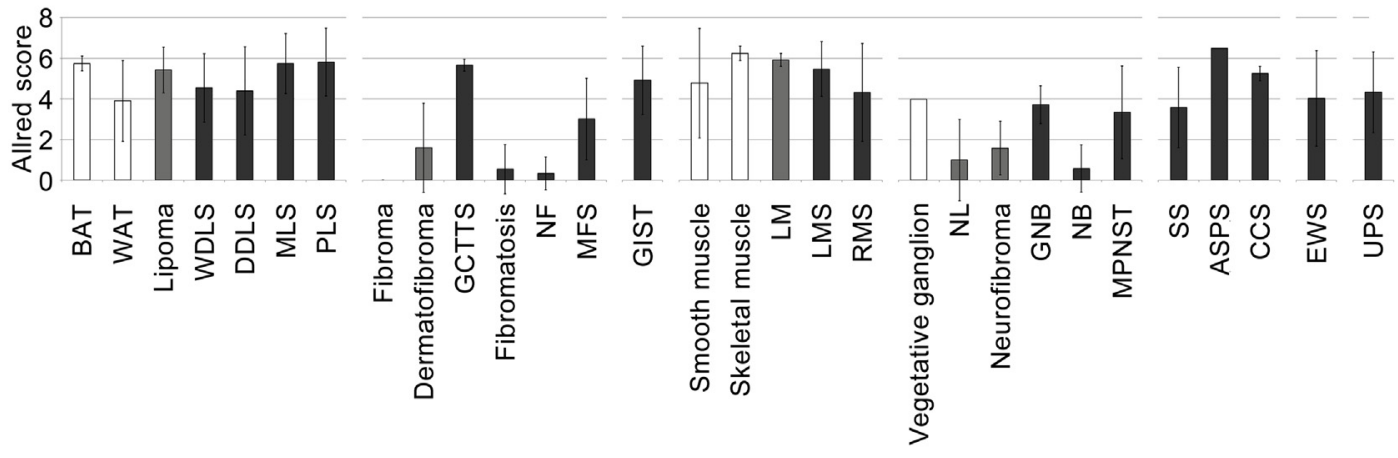

B
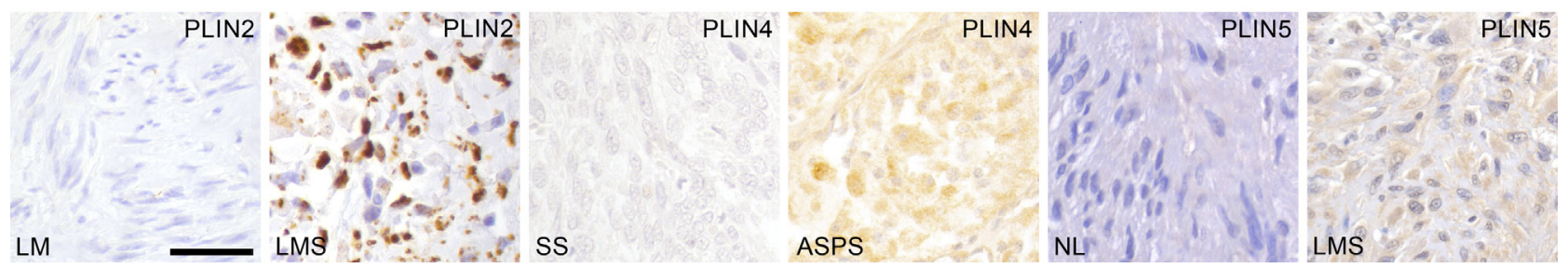

Figure 3 Perilipin (PLIN) expression in mesenchymal cells, benign soft tissue tumors, and sarcomas. A: Perilipin 1, 2, 4, and 5 staining (Supplemental Table S1) was scored according to staining intensity and percentage of cells using the Allred score: scores ranged from 0 (negative) to 8 (strong positivity in most cells). B: Representative examples of both positive and negative staining for perilipins 2, 4, and 5. Data are expressed as means \pm SD. Scale bar $=$ $50 \mu \mathrm{m}$. ASPS, alveolar soft part sarcoma; BAT, brown adipose tissue; CCS, clear cell sarcoma; DDLS, dedifferentiated liposarcoma; EWS, Ewing sarcoma; GCTTS, giant cell tumor of tendon sheath; GIST, gastrointestinal tract stroma tumor; GNB, ganglioneuroblastoma; LM, leiomyoma; LMS, leiomyosarcoma; MFS, myxofibrosarcoma; MLS, myxoid liposarcoma; MPNST, malignant peripheral nerve sheath tumor; NB, neuroblastoma; NF, nodular fasciitis; NL, neurilemmoma (schwannoma); PLS, pleomorphic liposarcoma; RMS, rhabdomyosarcoma; SS, synovial sarcoma; UPS, undifferentiated pleomorphic sarcoma; WAT, white adipose tissue; WDLS, well-differentiated liposarcoma.

perilipin 2 when compared with increasing grade of leiomyosarcomas or rhabdomyosarcomas (Figure $3 \mathrm{~B}$ and Supplemental Figure S4). Perilipin 3 expression showed a similar trend as perilipin 2, with higher expression in sarcomas, especially of higher grade when compared with benign tumors or normal tissues (Supplemental Figure S4). In some cases, however, increased perilipin 2 and 3 expression could be detected in the respective normal cells 
because of inflammatory infiltrates with foam cell macrophages or ischemic conditions. Perilipin 4 showed a similar expression pattern to perilipin 1, with a high expression in adipocytic tumors. Yet, perilipin 4 was also highly expressed in smooth and skeletal muscle tumors and alveolar soft part sarcomas, whereas Ewing sarcomas, synovial sarcomas, nerve sheath tumors, gastrointestinal tract stroma tumors, and fibroblastic/myofibroblastic tumors showed only minimal expression (Figure 3B). Likewise, perilipin 5 showed the highest expression in adipocytic and muscle tumors and the lowest expression in neural and fibroblastic/myofibroblastic tumors. Concerning the tumor grade, perilipin 2 and 3 showed a tendency to be higher expressed in sarcomas of higher grade (concerning leiomyosarcomas) (Supplemental Table S3) and for sarcomas in general (Supplemental Table S2). In contrast, perilipin 1 and 4 and, to a certain degree, also perilipin 5 showed higher expression in normal tissues or benign tumors and lower expression in sarcomas of higher grade.

In summary, LD accumulation, as demonstrated by perilipin staining, is present in virtually all sarcomas and not restricted to liposarcomas. Only perilipin 1 positivity at LDs differentiates liposarcomas from other soft tissue sarcomas. Perilipins 2, 3, 4, and 5 show wider expression among soft tissue tumors (eg, perilipin 4 and 5 expression is especially prominent in adipocytic and muscle tumors). Interestingly, perilipin 2 and 3 expression was often much higher in sarcomas when compared with the respective benign tumors. Perilipin 2 and 3 were often stronger in high-grade tumors compared with low-grade tumors as well (Supplemental Tables S1-S3 and Supplemental Figure S4).

\section{In Vitro Stimulation of Adipogenesis in Liposarcoma Cells}

To analyze the interplay between LDs and perilipins in a dynamic liposarcoma cell culture model, the LiSa-2 cell line ${ }^{26}$ was used and compared with PLS tissue, from which the cell line has been derived. Immunofluorescence analysis of PLS tissue revealed perilipin 1- and perilipin 2-positive LDs (BODIPY); however, the perilipin 1 staining pattern was heterogeneous with respect to LD subpopulations. Perilipins 1 and 2 localized to different LDs without any colocalization (Figure 4A).

Under normal culture conditions, LiSa-2 cells express only perilipins 2 and 3, whereas perilipins 1,4 , and 5 were negative. This is in contrast to the PLS tissue from which the cell line has been derived. Therefore, whether adipogenic differentiation may be regained in LiSa- 2 cells was tested. Adipogenesis was induced in LiSa-2 cells and the expression of perilipins was assayed over time up to 40 days. On day 0, control cells cultured with RPMI 1640 medium showed strong expression for perilipins 2 and 3, but no expression for perilipins 1, 4, and 5. Long-term culture of cells for up to 40 days led to increasing expression of perilipins 1,4 , and 5 , whereas perilipin 2 and 3 expression remained unchanged (Figure 4B). Adipogenic differentiation medium (preadipocyte/adipocyte differentiation medium supplemented with dimethyl sulfoxide) induced stronger perilipin 4 and reduced perilipin 3 expression in LiSa-2 cells (Figure 4B) compared with LiSa2 cells cultured in control medium (Figure 4B). Immunofluorescence staining of long-term cultured LiSa-2 cells further revealed a differential perilipin 1 expression compared with untreated cells on day 0. Perilipin 1 was completely absent on day 0 , whereas LD-bound perilipin 1 was found on day 40, independent of the medium used for differentiation. LDs were increased in size when LiSa-2 cells were treated with PAM-dimethyl sulfoxide medium compared with RPMI 1640 medium (Figure 4C).

\section{Silencing of Perilipins 2 and 3 in Cultured Liposarcoma Cells}

Because an increase was detected in perilipin 2 and 3 expression in sarcoma compared with benign soft tissue tumors or the respective normal cell type of origin, the usefulness of perilipin 2 and perilipin 3 as potential therapeutic targets in liposarcoma cells was evaluated. To determine whether an alteration of the tumor lipid metabolism may be functionally relevant, perilipin 2 and 3 expression in LiSa-2 cells was silenced using siRNA transfection. The knockdown was performed using two different siRNAs targeting perilipin 2, two siRNAs targeting perilipin 3, and three nontargeting siRNAs. Immunoblot results confirmed that the expression of perilipins 2 and 3 was sufficiently reduced on knockdown and that the expression of perilipins 1 and 4 remained unchanged (Figure 5A). The reduction in perilipin 2 and 3 expression was accompanied by a slightly reduced LD number that appeared to be larger in size because it contained more lipid per droplet. The effect appeared to be the highest in cells transfected with siRNAs against both perilipins at the same time (Figure 5B). Furthermore, cell viability was mildly reduced on siRNA-mediated knockdown of perilipin 2 or 3 (Figure 5C) and showed the strongest reduction of cells that showed a reduced perilipin 2 expression. The experiment was repeated with different adipocytic tumor cell lines, such as T778, a DDLS cell line (Supplemental Figure S5), and with different numbers of cells seeded (data not shown) showing comparable results.

In summary, the down-regulation of perilipins 2 and 3 showed a modest impact on cell viability and altered LD morphology. A therapeutic strategy may use this as a target.

\section{Discussion}

Analysis of LD-associated proteins in different types of liposarcomas and other types of soft tissue sarcomas revealed perilipin 1 as a promising marker for the differential diagnosis of liposarcomas from other, especially high-grade soft tissue sarcomas. Although there is marked LD accumulation 
A
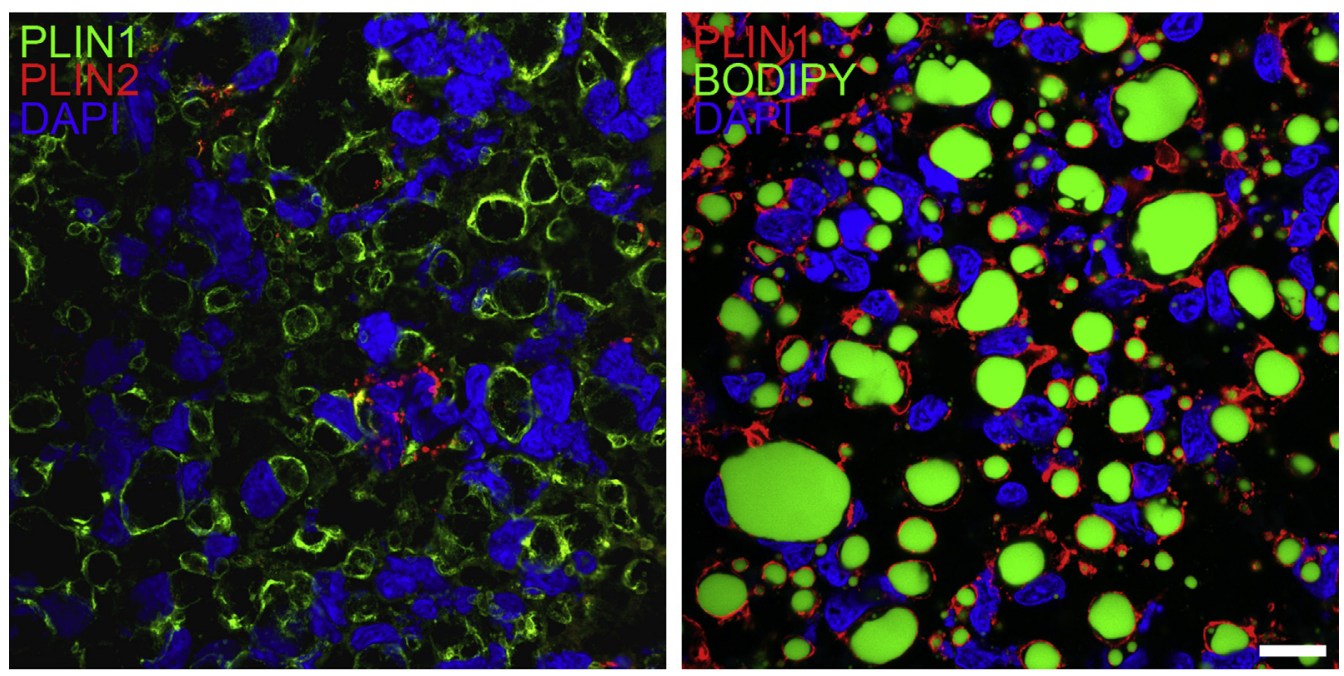

B

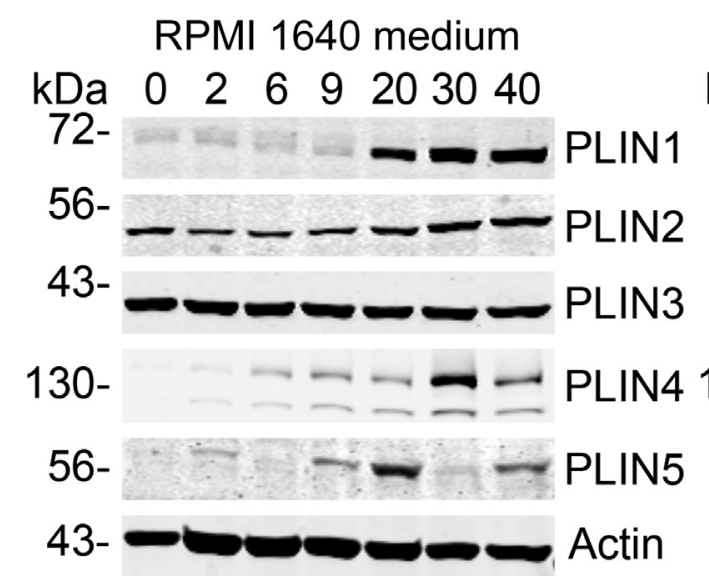

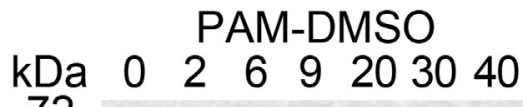

$72--2=-\cdots-$ PLIN1

56- - - - - PLIN2

43- - - - - PLIN3

130- ー밈INA

56- - - - - PLIN5

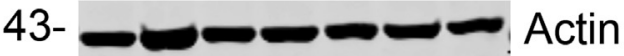

C

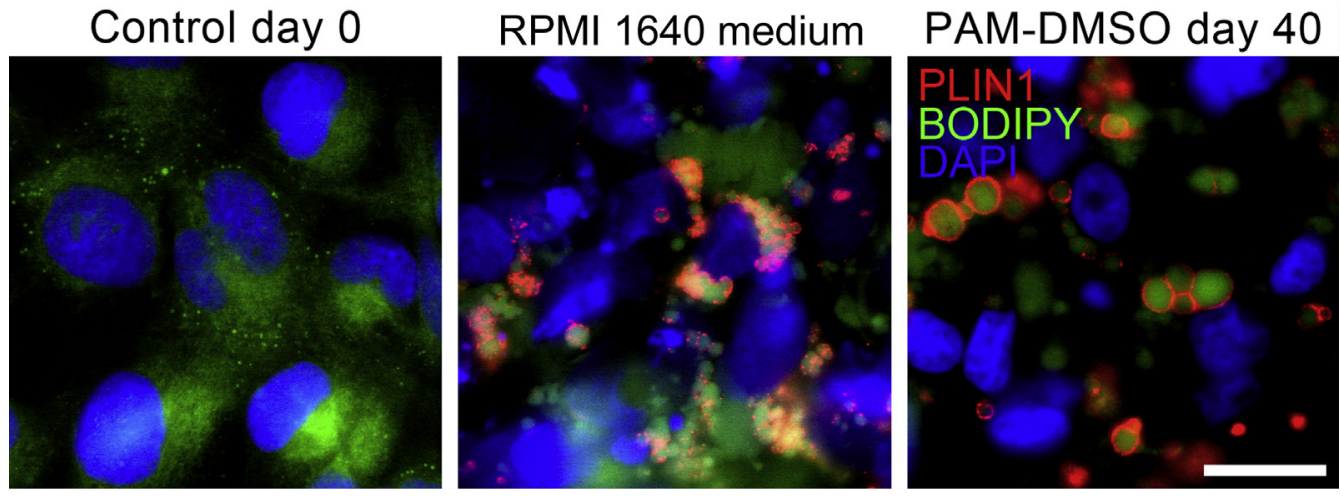

Figure 4 Induction of adipogenic differentiation in LiSa-2 cells. A: Immunofluorescence microscopy of the pleomorphic liposarcoma, from which the LiSa-2 cell line has been derived, with antibodies against perilipin 1 (PLIN1) and perilipin 2 (PLIN2). Lipid droplets (LDs) were stained with boron dipyrromethene (BODIPY), and nuclei were stained with DAPI. B: Immunoblot of whole cell lysates obtained from LiSa-2 cells cultured for 40 days with RPMI 1640 medium (left panel) or preadipocyte/adipocyte differentiation medium (PAM) supplemented with dimethyl sulfoxide (DMSO; right panel) stained with antibodies against PLIN1 through PLIN5. Samples were collected on days 0, 2, 6, 9, 20, 30, and 40. Actin served as the protein control. Molecular weight markers are shown on the left. C: Immunofluorescence analysis of cells on day 0 and cells cultured in either RPMI 1640 medium or PAMDMSO differentiation medium long-term (40 days). LiSa-2 cells were stained with antibodies against PLIN1. LDs were stained with B0DIPY, and nuclei were stained with DAPI. Scale bar $=20 \mu \mathrm{m}$. 

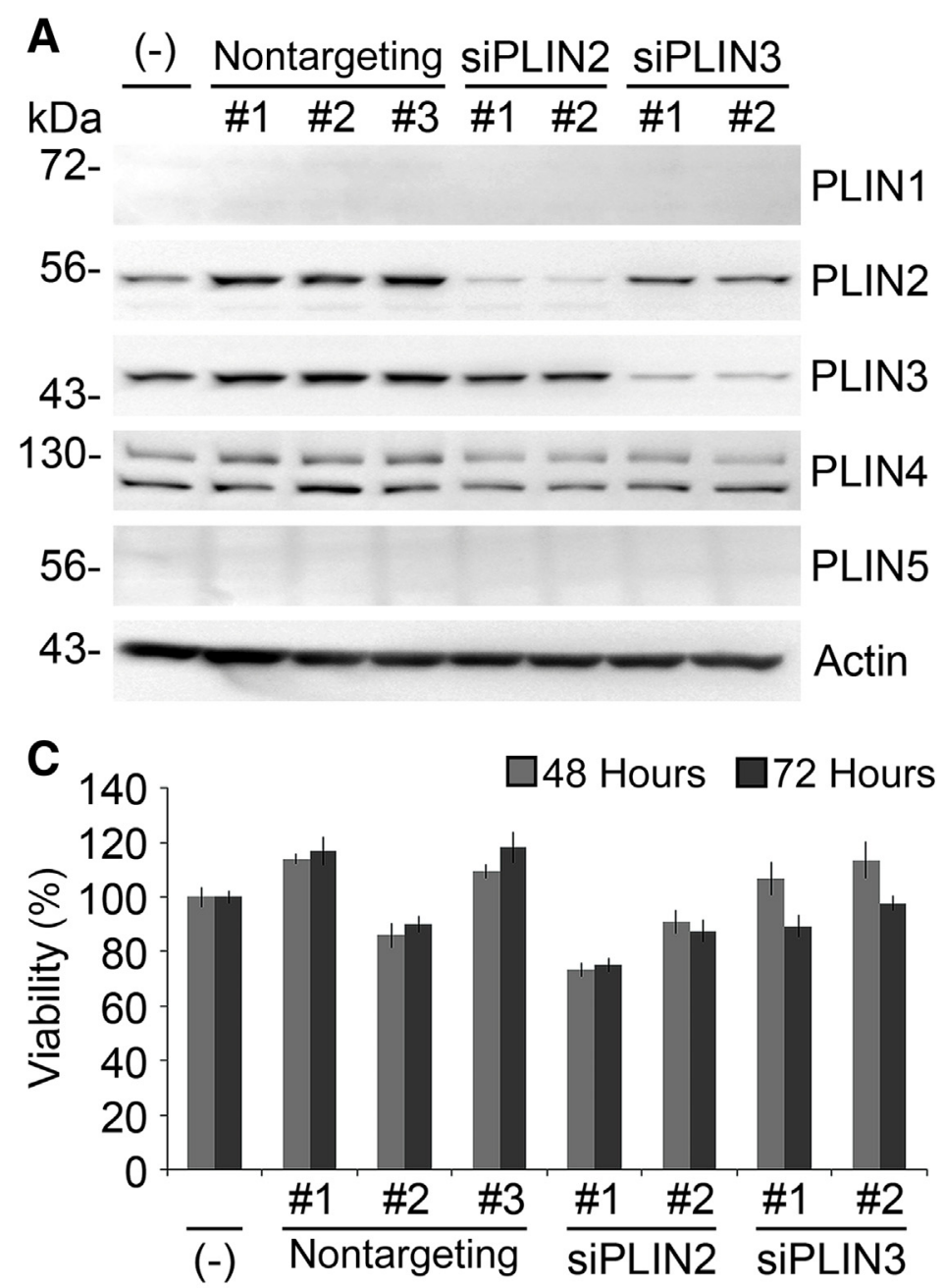
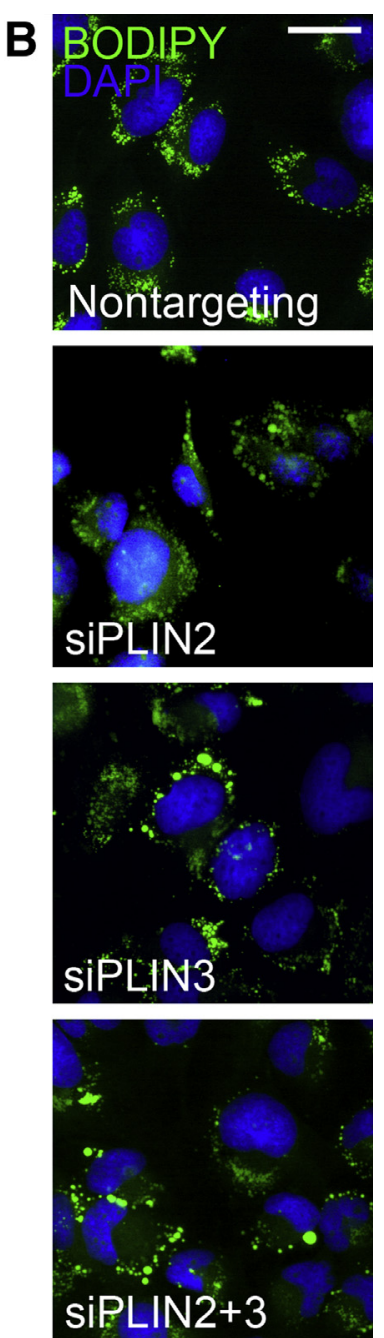

Figure 5 siRNA-mediated knockdown of perilipin 2 (siPLIN2) and/or 3 (siPLIN3) in LiSa-2 cells. A: Immunoblot of whole cell lysates obtained from either LiSa-2 cells transfected with two different siRNAs targeting perilipin 2, two siRNAs targeting perilipin 3, or three nontargeting siRNAs or nontransfected cells stained with antibodies against perilipins 1 through 5 (PLIN1-5). Actin served as the protein control. Molecular weight markers are shown on the left. B: Immunofluorescence analysis of LiSa-2 cells transfected with siRNAs targeting either perilipin 2 or perilipin 3, either together or with nontargeting siRNAs. Lipid droplets were stained with boron dipyrromethene (BODIPY), and nuclei were stained with DAPI. C: Cell viability assay (MTT assay) of either LiSa-2 cells transfected with two different siRNAs targeting perilipin 2, two siRNAs targeting perilipin 3, or three nontargeting siRNAs or nontransfected cells. Samples were collected and analyzed after 48 and 72 hours. Data are expressed as means \pm SD. $n=6$ technical replicates (C). Scale bar $=20 \mu \mathrm{m}(\mathbf{B})$.

accompanied by the expression of perilipins 2 and 3 as a frequent feature of most sarcomas, only perilipin 1 is expressed specifically in liposarcomas but not in other sarcomas. In nonneoplastic human tissues, perilipin 1 has been shown to be restricted to adipocytes of white and brown adipose tissue, to sebaceocytes, and to hepatocytes with longstanding fatty change, whereas it was absent from normal hepatocytes. ${ }^{14,31,33}$ Perilipin 1 is expressed in approximately one-third of hepatocellular carcinomas, ${ }^{32}$ in sebaceous carcinomas, ${ }^{32,34,35}$ and in liposarcomas, ${ }^{24,25}$ paralleling its expression in nonneoplastic tissues. All other tumors analyzed (sarcomas and carcinomas) were negative for perilipin 1 expression at LDs. ${ }^{32}$ The presence of LDs may thereby not be a prerequisite for liposarcoma or lipomatous differentiation. Only perilipin 1 positivity at LDs differentiates liposarcomas from other soft tissue sarcomas and may thereby be of use for histopathologic diagnosis. Metastases from hepatocellular carcinoma or sebaceous carcinoma that also express perilipin 1 can easily be identified because of their positivity for epithelial markers, such as keratins, and based on this pose no confusion in the diagnosis of liposarcoma. Perilipin 1 may supplement further markers, such as MDM2 and cyclin-dependent kinase $4,{ }^{36}$ in WDLSs or DDLSs, or even in absence of MDM2 amplification, as in the case of PLSs, where MDM2 amplification does not occur and S100 protein expression is only observed in less than half of the cases. Perilipin 1 may complement the set of currently used immunohistochemical markers together with S100 protein and CD34 in the diagnosis of MLS, especially in cases in which morphology alone may not be sufficient for proper diagnostics or genetic analysis for the diagnostic hallmarks of MLS as DDIT3 (CHOP) and FUS (TLS) fusion 
is not available. Perilipins 1 and 4 are differentiation markers, which are often lost in DDLS or PLS (Table 1). However, although not a highly sensitive marker, perilipin 1 is highly specific, so negativity for perilipin 1 does not necessarily exclude especially a diagnosis of DDLS or PLS. The possibility of a higher percentage of perilipin 1-positive tumors compared with the one given, when using full tumor sections instead of a tissue microarray, cannot be excluded. In hepatocellular tumors, perilipin 1 correlated negatively with tumor grade and with proliferation rate, as measured by Ki-67. ${ }^{32}$ Our study is keeping with the studies of Westhoff et $\mathrm{al}^{24}$ and Iwasaki et $\mathrm{al}^{25}$ who examined a small cohort of cases and previously proposed perilipin 1 as a marker for tumors of adipocytic origin. In contrary, cultured liposarcoma cells, such as LiSa-2 cells, derived from PLS lose perilipin 1 expression during culture. This has been previously observed for other mesenchymal cells, such as adipose tissue-derived mesenchymal stem cells, ${ }^{37}$ and perilipin 1 expression may only be regained after incubation with specific adipogenic differentiation media. We could show that the re-establishment of adipogenesis in the liposarcoma cell line LiSa-2 strongly alters perilipin expression in a differential manner. Furthermore, the uptake of fatty acids and the presence of LDs in liposarcomas may depend on the metabolic status of the patient. Thus, our results show that perilipin 1 is a specific marker of adipogenic differentiation. However, perilipin 1 does not seem to be an adequate therapeutic target, as it is dispensable, as demonstrated in the respective cell cultures (compare with two other studies ${ }^{18,19}$ ).

In contrast to perilipin 1, which is almost exclusively restricted to adipocytic tumors, perilipins 2 through 5 are expressed in virtually all soft tissue tumors, and often in astonishingly high amounts. Perilipins 4 and $5^{29}$ are most prominently expressed in adipocytic tumors, whereas only perilipin 4 shows a similar expression pattern to perilipin 1 within this group of tumors. In addition, perilipin 5 is also expressed in rhabdomyomatous and leiomyomatous tumors and the respective normal cells. Perilipins 2 and 3, however, are positive in virtually all sarcomas but prominently in high-grade sarcomas, only faintly expressed in welldifferentiated liposarcomas, and negative in lipomas and in WAT (for utility of perilipin 2 in PLS ${ }^{38}$ ). Therefore, in our hands, perilipins 2 and 3 are not markers of choice to clearly differentiate well-differentiated liposarcoma from lipoma. We have previously shown that in tumorigenesis, especially in high-grade tumors, LD accumulation, together with an increased perilipin 2 and 3 expression, is frequently observed. This is the case in hepatocellular carcinoma and breast cancer, where LD accumulation correlates with the Ki67 proliferation rate, ${ }^{32}$ that is likely a result of an altered tumor metabolism also commonly known as the Warburg effect. ${ }^{39,40}$ Multiple studies have shown that both enhanced glycogenesis and enhanced lipogenesis are hallmarks of cancer and especially for liposarcoma ${ }^{41}$ and may be therapeutically amenable. In addition, in cultured PLS-derived LiSa-2 cells and DDLS-derived T778 cells, vitality was slightly reduced when perilipin 2 and/or perilipin 3 was reduced via siRNA, although the number of LDs and triacyl glycerol levels were only mildly reduced because of a compensatory increase in LD size. Similarly, siRNAmediated down-regulation of perilipins 2 and 3 in hepatoma cells (Huh7) leads to a reduced number of LDs with increased size and to concomitant reduced cell vitality. ${ }^{31}$ LDs and in particular LD-associated perilipins 2 and 3 may be interesting therapeutic targets in malignant tumors, carcinoma, and sarcoma. As previously shown for the inhibition of the fatty acid synthase, ${ }^{42}$ targeting lipid metabolism in liposarcoma may be a good approach in terms of developing proper therapeutic strategies. Such therapies might be extended by specifically targeting perilipins 2 and 3, whereas perilipin 1 expression may be used to improve proper diagnosis.

\section{Acknowledgments}

We thank Elisabeth Specht-Delius and Marion Moock for expert technical assistance; Ronald Unger and Sonja Witzel for critical reading of the manuscript and helpful discussions; the tissue bank of the National Center for Tumor Diseases (Heidelberg, Germany) for providing all human tissue specimens; and the Nikon Imaging Facility (Heidelberg, Germany) for performing confocal laser scanning microscopy.

B.K.S. is the guarantor of this work, has full access to all of the data in the study, and takes responsibility for the integrity of the data and the accuracy of the data analysis.

\section{Supplemental Data}

Supplemental material for this article can be found at http://doi.org/10.1016/j.ajpath.2019.04.017.

\section{References}

1. Kimmel AR, Brasaemle DL, McAndrews-Hill M, Sztalryd C, Londos C: Adoption of PERILIPIN as a unifying nomenclature for the mammalian PAT-family of intracellular lipid storage droplet proteins. J Lipid Res 2010, 51:468-471

2. Greenberg AS, Egan JJ, Wek SA, Garty NB, Blanchette-Mackie EJ, Londos C: Perilipin, a major hormonally regulated adipocyte-specific phosphoprotein associated with the periphery of lipid storage droplets. J Biol Chem 1991, 266:11341-11346

3. Brasaemle DL, Barber T, Wolins NE, Serrero G, BlanchetteMackie EJ, Londos C: Adipose differentiation-related protein is an ubiquitously expressed lipid storage droplet-associated protein. J Lipid Res 1997, 38:2249-2263

4. Heid HW, Moll R, Schwetlick I, Rackwitz HR, Keenan TW: Adipophilin is a specific marker of lipid accumulation in diverse cell types and diseases. Cell Tissue Res 1998, 294:309-321

5. Jiang HP, Serrero G: Isolation and characterization of a full-length cDNA coding for an adipose differentiation-related protein. Proc Natl Acad Sci U S A 1992, 89:7856-7860

6. Diaz E, Pfeffer SR: TIP47: a cargo selection device for mannose 6phosphate receptor trafficking. Cell 1998, 93:433-443

7. Wolins NE, Rubin B, Brasaemle DL: TIP47 associates with lipid droplets. J Biol Chem 2001, 276:5101-5108 
8. Wolins NE, Skinner JR, Schoenfish MJ, Tzekov A, Bensch KG, Bickel PE: Adipocyte protein S3-12 coats nascent lipid droplets. J Biol Chem 2003, 278:37713-37721

9. Wolins NE, Brasaemle DL, Bickel PE: A proposed model of fat packaging by exchangeable lipid droplet proteins. FEBS Lett 2006 , 580:5484-5491

10. Yamaguchi T, Matsushita S, Motojima K, Hirose F, Osumi T: MLDP, a novel PAT family protein localized to lipid droplets and enriched in the heart, is regulated by peroxisome proliferatoractivated receptor alpha. J Biol Chem 2006, 281:14232-14240

11. Kassan A, Herms A, Fernandez-Vidal A, Bosch M, Schieber NL, Reddy BJ, Fajardo A, Gelabert-Baldrich M, Tebar F, Enrich C, Gross SP, Parton RG, Pol A: Acyl-CoA synthetase 3 promotes lipid droplet biogenesis in ER microdomains. J Cell Biol 2013, 203:985-1001

12. Wolins NE, Quaynor BK, Skinner JR, Schoenfish MJ, Tzekov A, Bickel PE: S3-12, adipophilin, and TIP47 package lipid in adipocytes. J Biol Chem 2005, 280:19146-19155

13. Takahashi Y, Shinoda A, Inoue J, Sato R: The gene expression of the myocardial lipid droplet protein is highly regulated by PPARgamma in adipocytes differentiated from MEFs or SVCs. Biochem Biophys Res Commun 2010, 399:209-214

14. Straub BK, Stoeffel P, Heid H, Zimbelmann R, Schirmacher P: Differential pattern of lipid droplet-associated proteins and de novo perilipin expression in hepatocyte steatogenesis. Hepatology 2008, 47:1936-1946

15. Arimura N, Horiba T, Imagawa M, Shimizu M, Sato R: The peroxisome proliferator-activated receptor gamma regulates expression of the perilipin gene in adipocytes. J Biol Chem 2004, 279:10070-10076

16. Dalen KT, Schoonjans K, Ulven SM, Weedon-Fekjaer MS, Bentzen TG, Koutnikova H, Auwerx J, Nebb HI: Adipose tissue expression of the lipid droplet-associating proteins S3-12 and perilipin is controlled by peroxisome proliferator-activated receptorgamma. Diabetes 2004, 53:1243-1252

17. Martinez-Botas J, Anderson JB, Tessier D, Lapillonne A, Chang BH, Quast MJ, Gorenstein D, Chen KH, Chan L: Absence of perilipin results in leanness and reverses obesity in $\operatorname{Lepr}(\mathrm{db} / \mathrm{db})$ mice. Nat Genet 2000, 26:474-479

18. Tansey JT, Sztalryd C, Gruia-Gray J, Roush DL, Zee JV, Gavrilova O, Reitman ML, Deng CX, Li C, Kimmel AR, Londos C: Perilipin ablation results in a lean mouse with aberrant adipocyte lipolysis, enhanced leptin production, and resistance to diet-induced obesity. Proc Natl Acad Sci U S A 2001, 98:6494-6499

19. Gandotra S, Le Dour C, Bottomley W, Cervera P, Giral P, Reznik Y, Charpentier G, Auclair M, Delepine M, Barroso I, Semple RK, Lathrop M, Lascols O, Capeau J, O'Rahilly S, Magre J, Savage DB, Vigouroux C: Perilipin deficiency and autosomal dominant partial lipodystrophy. N Engl J Med 2011, 364:740-748

20. Granneman JG, Moore HP, Mottillo EP, Zhu Z, Zhou L: Interactions of perilipin-5 (Plin5) with adipose triglyceride lipase. J Biol Chem 2011, 286:5126-5135

21. Wang H, Sztalryd C: Oxidative tissue: perilipin 5 links storage with the furnace. Trends Endocrinol Metab 2011, 22:197-203

22. Scherer PE, Bickel PE, Kotler M, Lodish HF: Cloning of cell-specific secreted and surface proteins by subtractive antibody screening. Nat Biotechnol 1998, 16:581-586

23. Russeler AV, Brors B, Fischer T, Hartmann JT, Hartmann W, Hohenberger P, Lichter P, Marx A, Mechtersheimer G, Penzel R, Renner M, Schildhaus HU, Schirmacher P, Sievers E, Strobel P, Wardelmann E, Ziesche E, Buttner R: [Molecular pathology of sarcomas: update on the research group "molecular diagnosis of sarcomas"] German. Pathologe 2010, 31 Suppl 2:211-214

24. Westhoff CC, Mrozinski J, Riedel I, Heid HW, Moll R: Perilipin 1 is a highly specific marker for adipocytic differentiation in sarcomas with intermediate sensitivity. J Cancer Res Clin Oncol 2017, 143:225-232

25. Iwasaki H, Ishiguro M, Nishio J, Aoki M, Yokoyama R, Yokoyama K, Taguchi K, Nabeshima K: Extensive lipoma-like changes of myxoid liposarcoma: morphologic, immunohistochemical, and molecular cytogenetic analyses. Virchows Arch 2015, 466:453-464

26. Wabitsch M, Bruderlein S, Melzner I, Braun M, Mechtersheimer G, Moller P: LiSa-2, a novel human liposarcoma cell line with a high capacity for terminal adipose differentiation. Int J Cancer 2000, 88:889-894

27. Koelsche C, Renner M, Hartmann W, Brandt R, Lehner B, Waldburger N, Alldinger I, Schmitt T, Egerer G, Penzel R, Wardelmann E, Schirmacher P, von Deimling A, Mechtersheimer G: TERT promoter hotspot mutations are recurrent in myxoid liposarcomas but rare in other soft tissue sarcoma entities. J Exp Clin Cancer Res 2014, 33:33

28. Pawella LM, Hashani M, Schirmacher P, Straub BK: [Lipid dropletassociated proteins in steatosis: effects of induction and siRNAmediated downregulation of PAT proteins in cell culture models of hepatocyte steatosis] German. Pathologe 2010, 31 Suppl 2:126-131

29. Hashani M, Witzel HR, Pawella LM, Lehmann-Koch J, Schumacher J, Mechtersheimer G, Schnolzer M, Schirmacher P, Roth W, Straub BK: Widespread expression of perilipin 5 in normal human tissues and in diseases is restricted to distinct lipid droplet subpopulations. Cell Tissue Res 2018, 374:121-136

30. Straub BK, Rickelt S, Zimbelmann R, Grund C, Kuhn C, Iken M, Ott M, Schirmacher P, Franke WW: E-N-cadherin heterodimers define novel adherens junctions connecting endoderm-derived cells. J Cell Biol 2011, 195:873-887

31. Pawella LM, Hashani M, Eiteneuer E, Renner M, Bartenschlager R, Schirmacher P, Straub BK: Perilipin discerns chronic from acute hepatocellular steatosis. J Hepatol 2014, 60:633-642

32. Straub BK, Herpel E, Singer S, Zimbelmann R, Breuhahn K, MacherGoeppinger S, Warth A, Lehmann-Koch J, Longerich T, Heid H, Schirmacher P: Lipid droplet-associated PAT-proteins show frequent and differential expression in neoplastic steatogenesis. Mod Pathol 2010, 23:480-492

33. Straub BK, Gyoengyoesi B, Koenig M, Hashani M, Pawella LM, Herpel E, Mueller W, Macher-Goeppinger S, Heid H, Schirmacher P: Adipophilin/perilipin-2 as a lipid droplet-specific marker for metabolically active cells and diseases associated with metabolic dysregulation. Histopathology 2013, 62:617-631

34. Muthusamy K, Halbert G, Roberts F: Immunohistochemical staining for adipophilin, perilipin and TIP47. J Clin Pathol 2006, 59:1166-1170

35. Dozsa A, Dezso B, Toth BI, Bacsi A, Poliska S, Camera E, Picardo M, Zouboulis CC, Biro T, Schmitz G, Liebisch G, Ruhl R, Remenyik E, Nagy L: PPARgamma-mediated and arachidonic aciddependent signaling is involved in differentiation and lipid production of human sebocytes. J Invest Dermatol 2014, 134:910-920

36. Pilotti S, Della Torre G, Lavarino C, Sozzi G, Minoletti F, Vergani B, Azzarelli A, Rilke F, Pierotti MA: Molecular abnormalities in liposarcoma: role of MDM2 and CDK4-containing amplicons at 12q1322. J Pathol 1998, 185:188-190

37. Heid H, Rickelt S, Zimbelmann R, Winter S, Schumacher H, Dorflinger Y, Kuhn C, Franke WW: On the formation of lipid droplets in human adipocytes: the organization of the perilipinvimentin cortex. PLoS One 2014, 9:e90386

38. Ramirez-Bellver JL, Lopez J, Macias E, Alegria-Landa V, Gimeno I, Perez-Plaza A, Kutzner H, Requena L: Primary dermal pleomorphic liposarcoma: utility of adipophilin and MDM2/CDK4 immunostainings. J Cutan Pathol 2017, 44:283-288

39. Warburg O: On the origin of cancer cells. Science 1956, 123:309-314

40. Vander Heiden MG, Cantley LC, Thompson CB: Understanding the Warburg effect: the metabolic requirements of cell proliferation. Science 2009, 324:1029-1033

41. Zaidi N, Lupien L, Kuemmerle NB, Kinlaw WB, Swinnen JV, Smans K: Lipogenesis and lipolysis: the pathways exploited by the cancer cells to acquire fatty acids. Prog Lipid Res 2013, 52:585-589

42. Hughes DT, Martel PM, Kinlaw WB, Eisenberg BL: The synthetic triterpenoid CDDO-Im inhibits fatty acid synthase expression and has antiproliferative and proapoptotic effects in human liposarcoma cells. Cancer Invest 2008, 26:118-127 